\title{
Cost of Illness Analysis of Knee Osteoarthritis in a Tertiary Care Hospital
}

\author{
Saida Firdose Venkatagiri Shaik, Dhivya Kothandan*, Pravallika Singareddy, Santhosh Sanku \\ Department of Pharmacy Practice, Sri Venkateswara College of Pharmacy, Chittoor, Andhra Pradesh, INDIA.
}

\begin{abstract}
Background: Osteoarthritis $(O A)$ is the most common rheumatologic problem affecting people all over the world. The economic impact of OA is significant due to the effect of disability, co morbid disease and the expense of treatment. Since the economic burden of OA increases every year, this study was conducted to analyse the cost of illness (healthcare cost) of knee OA by calculating the direct and indirect costs. Methods: This was a prospective observational study conducted in the Orthopedics department of Tertiary Care Hospital at Chittoor District for a period of 4 months (September 2017 - December 2017). OA patients of age greater than 40 years, either gender who stayed in hospital and out-patients were included in the study.All the relevant and necessary data of OA patients were collected and total direct as well as indirect costs was calculated by human capital method. Results: A total of 160 patients were enrolled during the study period. Out of 160 patients, 38 (23.7\%) were male and 122 (76.3\%) were female. The average total direct cost per OA patient was found to be INR 11521 whereas indirect
\end{abstract}

cost per OA patient was INR 8998. The sum of total direct cost and indirect cost was INR 20519. Conclusion: The cost estimates of our study were significant and found mainly due to direct costs though it is not statistically significant. This report may helpful in conducting future studies of economic evaluations and outcomes research.

Key words: Cost of illness, Economic burden, Osteoarthritis, Co-morbidity. Correspondence

Dhivya Kothandan, Department of Pharmacy Practice, Sri Venkateswara College of Pharmacy, Chittoor - 517 127, INDIA.

Phone: 9626918783

Email: divyapharmd@gmail.com

DOI: 10.5530/jyp.2018.10.71

\section{INTRODUCTION}

Osteoarthritis (OA) is a common form of arthritis and one of the major public health problems worldwide. It is most commonly seen in elderly people and considered as a leading cause of chronic pain and disability by primarily affecting large weight bearing joints (hip, knees, spine), non-weight bearing joints (hands- fingers and thumb)and other joints (shoulder, elbow, ankle). ${ }^{1}$ OA also known as degenerative arthritis or degenerative joint disease or osteoarthrosis is a musculoskeletal disorder leads to degeneration of joints, loss of articular cartilage, subchondral sclerosis, hypertrophy of bone at the margins, biochemical and morphological alterations of the synovial membrane and joint capsule. ${ }^{2} \mathrm{OA}$ is the second most common rheumatologic problem with a prevalence of $22 \%$ to $39 \%$ in India. ${ }^{3}$ Nearly $80 \%$ of elderly people suffers with OA, among that approximately $20 \%$ of patients reported to have severe knee pain and not able to coping with daily activities in India. ${ }^{4} \mathrm{Knee} \mathrm{OA}$ is found to be more common in women than men, often times at the age of 60 . The incidence of $\mathrm{OA}$ increases with age. Many studies showed that prevalence of OA step-up with age especially between 30-65 years and raises burden on health care..$^{5-6}$ The economic impact of $\mathrm{OA}$ is significant due to the effect of disability, comorbid disease and the expense of treatment. The number of people with OA disability is expected to double by the year 2020, thereby increasing the already significant economic burden of OA. ${ }^{7}$ The impact of OA on society is usually estimated by examining the costs. Though costs measured in different forms, generally measured in monetary terms. Cost of illness (COI) was the first economic evaluation technique in health care system which helps in estimating the disease impact on health outcomes in a country, specific regions, communities and even individuals. ${ }^{8} \mathrm{COI}$ generates cost resulting from an illness which includes direct cost (medical and non-medical) includes cost of medical and non-medical expenditures (medications, diagnostics, hospitalizations, assistive devices, transport and home adaptations etc.,), indirect cost represent lost resources (productivity lost) due to being ill and intangible cost. ${ }^{9-10}$ Most of the COI studies included only direct cost and excluded indirect cost as well as intangible cost. Usually, intangible cost is about the pain, grief and stress related to being ill which is not related to money and not measurable one. ${ }^{11-12}$ Therefore, it is excluded from our study too. Since the economic burden of OA increases every year, this study was conducted to analyse the healthcare cost of knee OA by calculating the direct and indirect costs.

\section{METHODOLOGY}

\section{Study Design and Setting}

This was a prospective observational study conducted in the Orthopedics department of Tertiary Care Hospital at Chittoor District for a period of 4 months (September - December 2017). The study protocol was approved by the institutional ethical committee (IEC/RVSIMS/2017/07). Consent from hospital authorities and rheumatologist were obtained before accessing data from the patients. Patients who fulfilled the inclusion criterion were documented from the case sheets and recorded in a separately designed case report form. Written informed consents were obtained after explaining the study protocol to each individual patient.

\section{Patient Recruitment}

OA patients of age greater than 40 years, either gender who stayed in hospital and out-patients were included in the study. Pregnant women and those who avail reimbursement were excluded. A total of 160 patients were identified during ward rounds and through regular case record reviews during the study period. 


\section{Data Collection}

The enrolled subjects were followed from the day of admission until the day of discharge. All the relevant and necessary data were collected from patient's medical records, pharmacy records and case sheets as well as by interviewing patients, patient care takers and health-care professionals.

\section{Estimation of Direct and Indirect Cost}

Total direct costs include direct medical and direct non-medical costs. The direct medical cost includes the costs of OA medications and other co morbid conditions along with administration charge, laboratory investigations, consultation, hospitalization, other hospital treatment and adjunctive therapy. The direct non-medical costs include the costs of transportation to the hospital, assistive devices and household changes. Total direct costs were calculated as the sum of medical and non-medical cost. Indirect costs were calculated as the sum of cost of productivity loss (patient and caregiver) and coping cost done by human capital method.

\section{RESULT}

A total of 160 patients were enrolled during the study period. Out of 160 patients, 38 (23.7\%) were male and $122(76.3 \%)$ were female. The other demographic details are summarized in Table 1.
The average total direct cost per OA patient was INR 11521. Out of this, the average medical cost was INR 8713 (76\%) and non-medical cost was INR 2808 (24\%). The average indirect cost per OA patient was INR 8998. The sum of total direct cost and indirect cost was INR 20519. The percentage of total direct cost was 56\% (medical - $42 \%$ and non-medical - $14 \%$ ) and indirect cost was $44 \%$.The various components included in calculating health care cost of knee OA are shown in Table 2 and 3.

The presence of statistically significant difference between direct and indirect cost was determined by means of unpaired t-test. The value of $p<0.05$ was considered statistically significant, since the confidence interval was maintained at $95 \%$.

Statistically significant difference was not found between direct and indirect costs $[P(0.1475)]$ as shown in Figure 1.

\section{DISCUSSION}

The economic burden of OA is increasing every year. This study has been carried to estimate the costs associated with OA and also to find out the factors which are significantly associated with increased cost of OA. This study has aggregated the data of 160 patients. The mean age of those included was 56 years which is similar to the report of Elena et al. study. ${ }^{13}$ This study has analyzed both direct and indirect costs

Table 1: Demographic details of the studied population ( $N=160)$.

\begin{tabular}{|c|c|c|c|}
\hline Characteristic & No. of Patients (\%) & Characteristic & No. of Patients (\%) \\
\hline Age groups(years) & & Allergy & \\
\hline $30-40$ & $3(1.85)$ & Yes & $154(96.3)$ \\
\hline $40-50$ & $37(23.13)$ & No & $6(3.7)$ \\
\hline $50-60$ & $54(33.8)$ & Social History & \\
\hline $60-70$ & $48(30)$ & Smokers/Alcoholic & $155(96.8)$ \\
\hline $70-80$ & $15(9.4)$ & Non-smokers/Non-alcoholic & $5(3.2)$ \\
\hline$>80$ & $3(1.85)$ & & \\
\hline Gender & & Substance Abuse & \\
\hline Males & $38(23.7)$ & Betel-nut and Betel leaf & $22(13.8)$ \\
\hline Females & $122(76.3)$ & No abuse & $138(86.2)$ \\
\hline Height $\left(\mathrm{m}^{2}\right)$ & $2.73 \pm 0.174$ & Surgical History & \\
\hline Weight $(\mathrm{kg})$ & $55.83 \pm 3.53$ & Yes & $59(37)$ \\
\hline Body Mass Index $\left(\mathrm{kg} / \mathrm{m}^{2}\right)$ & & No & $101(63)$ \\
\hline Under weight $(<18.5)$ & & Co-morbidities & \\
\hline Normal weight(18.5-25) & $10(6.3)$ & Hypertension & $39(24.4)$ \\
\hline Over weight(25-30) & $148(92.5)$ & Diabetes Mellitus & $49(31)$ \\
\hline Obese $(>30)$ & $1(0.6)$ & Asthma & $2(1.2)$ \\
\hline Literacy background & $1(0.6)$ & Ulcer & $2(1.2)$ \\
\hline Illiterate & & Duration of Osteoarthritis & \\
\hline Literate & $100(62.5)$ & 1-6 months & $137(85.6)$ \\
\hline Occupational Status & $60(37.5)$ & 6-12 months & $12(7.5)$ \\
\hline House wife & & $>1$ year & $7(4.4)$ \\
\hline Farmer & $77(48.1)$ & $>2$ years & $4(2.5)$ \\
\hline Employee & $58(36.3)$ & No of drugs per prescriptions & \\
\hline Business & $2(1.2)$ & $2-3$ & $39(24.4)$ \\
\hline Self employed & $10(6.3)$ & $4-6$ & $114(71.2)$ \\
\hline Labor & $4(2.5)$ & $7-10$ & $7(4.4)$ \\
\hline Retired & $5(3.1)$ & Length of hospital stay(Days) & \\
\hline Marital Status & $4(2.5)$ & 0 & $110(68.8)$ \\
\hline Married & & $1-3$ & $42(26.2)$ \\
\hline Single/Widow & $145(90.6)$ & $4-5$ & $8(5)$ \\
\hline Food Habit & $15(9.4)$ & & \\
\hline Vegetarian & $8(5)$ & & \\
\hline Non-vegetarian & $152(95)$ & & \\
\hline
\end{tabular}


Table 2: Components of Total Direct Cost.

\begin{tabular}{cccc}
\hline S. No & $\begin{array}{c}\text { Cost of Direct Cost } \\
\text { Components }\end{array}$ & $\begin{array}{c}\text { Average Cost } \\
\text { (INR) }\end{array}$ & $\%$ \\
\hline 1 & Investigation / lab tests & 471 & 4 \\
2 & Medications for osteoarthritis & 1014 & 9 \\
3 & Medications for co-morbid & 378 & 3 \\
& conditions & & 4 \\
4 & Hospitalization & 500 & 2 \\
5 & Doctor consultation & 255 & 1 \\
6 & Means of transport & 80 & 51 \\
7 & Visited other hospital & 5884 & 2 \\
8 & Adjunctive therapy & 211 & \\
& (Acupuncture /Physiotherapy/ & & 7 \\
9 & Massaging) & 778 & 17 \\
10 & Assistive device & 1950 & \\
\hline
\end{tabular}

Table 3: Components of Indirect Cost.

\begin{tabular}{cccc}
\hline S. No & $\begin{array}{c}\text { Cost of Indirect Cost } \\
\text { Components }\end{array}$ & $\begin{array}{c}\text { Average Cost } \\
\text { (INR) }\end{array}$ & $\%$ \\
\hline 1 & Patient's loss of productivity & 2290 & 25 \\
2 & Care giver's loss of productivity & 1430 & 16 \\
3 & Coping cost & 5278 & 59 \\
& Total Cost & 8998 & \\
\hline
\end{tabular}

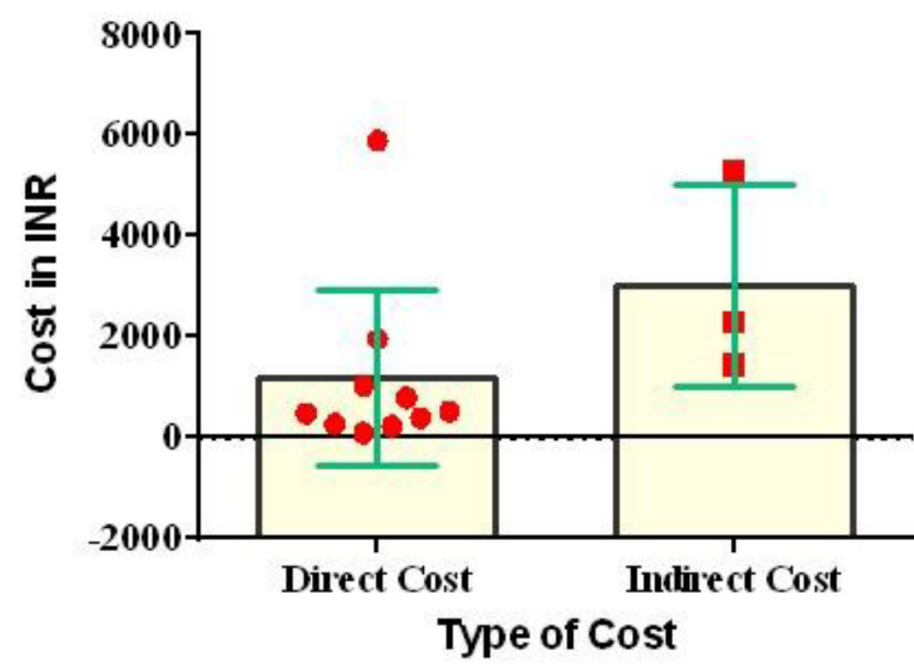

Figure 1: Comparison of Direct and Indirect Cost.

which were calculated by taking an average value of each component. The average direct cost of OA per patient was INR 11521 consisting of medical and non-medical costs which is similar to David et al. study. ${ }^{14}$ This study has evaluated the costs of 7 medical components (component with minimum cost was adjunctive therapy- INR 211 and component with maximum cost was visiting other hospital for treatment of OA -INR 5884) and 3 non-medical components (component with minimum cost was means of transport- INR 80 and component with maximum cost was household changes INR 1950) of total direct costs. ${ }^{15-16}$ Overall, means of transport was the cheapest item and visited other hospital for treating OA was an expensive item among total direct cost. With respect to the cost of co-morbidity, majority of our study population found with two common co-morbidities i.e., hypertension and diabetes mellitus (49 patients with diabetes mellitus and 39 patients with hypertension). ${ }^{17}$ The average cost spent for treating co-morbidities was INR 378. When analysing the hospitalization cost, our study has found that $69 \%$ of patients were outpatients so the average hospitalization cost was found to be less i.e., INR 500. ${ }^{18}$ In the analysis of OA medication cost, it should be noted that OA dugs were prescribed for only 15 days and therapy review was planned after 15 days so the cost spent for OA medications was found to be INR 1014which disagree with the result of Le TK et al. study. ${ }^{19}$ In terms of analyzing the cost spent for assistive device was INR 778. With regards to the consultation fee and investigation cost, it was INR 255 and INR 471which varies from the report of Logan et al study. ${ }^{20}$ The mean value of indirect cost per OA patient was INR 8998. Three components were included to analyze indirect costs. All - inclusive, caregiver's cost for their loss of productivity due to informal care was lowcost component (INR 1430) and coping cost (INR 5278) was high-cost component among indirect costs. Coping cost refers to borrowing, sale of assets, investing money from savings and reduction in consumption of food. Another important item was patient's loss of productivity caused by an illness was INR 2290. ${ }^{21}$ Adding together the costs of direct and indirect cost gives total cost which is needed to treat per patient with knee OA was analyzed to be INR 20519. Among the total costs, direct cost (56\%) was found to be more than indirect cost (44\%) but significant difference was not found statistically between these two costs with the $P$ value of 0.1475 which is shown in Figure 1 which agree to Chen et al. study. ${ }^{22}$

\section{CONCLUSION}

As far as we know, it was the first study conducted in assessing the health care costs of OA in India. The cost estimates of our study were significant and found mainly due to direct costs though it is not statistically significant. The economic burden of OA increases mainly due to direct costs. The data conferred from this study may helpful in conducting future studies of economic evaluations and outcomes research. The main limitations of this study were smaller sample size, duration of the study and performed in a single center. Multi-centre studies should be conducted to compare the cost of $\mathrm{OA}$ in future.

\section{ACKNOWLEDGEMENT}

The authors are thankful to the subjects who participated in the study and management of Sri Venkateswara College of Pharmacy for providing excellent research guidance and support.

\section{CONFLICT OF INTEREST}

The authors declare no conflict of interest.

\section{ABBREVIATIONS}

OA: Osteoarthritis; COI: Cost of Illness; INR: Indian Rupee.

\section{REFERENCES}

1. Marina CA, Sandra MB, Meira V, Elen G. Reflections about osteoarthritis and curcuma longa. Phcog Rev. 2017;11(21):8-12.

2. Mesfin $Y$, Young L, Ping J, Mei H, Jeong N, Lidia B, et al. UP 1306, a botanical composition with analgesic and anti-inflammatory effect. Phcog Res. 2016;8(3):186-92.

3. Mesfin Y, Young L, Meira V Elen G. Analgesic and anti-inflammatory effect of UP3005, botanical composition containing two standardized extracts of uncariagambir and morusalba. Phcog Res. 2015;7(1):39-46.

4. Viswanath J, Chakrapani C, Renu D, Sankaraiah S. Prevalence of osteoarthritis patients in south Indian hospital. Int J Community Medand Public Health. 
2017;4(8):3043-46

5. Hinman RS, Hunt MA, Creaby MW, Wrigley T, McManus FJ, Bennell KL. Hip muscle weakness in individuals with medial knee osteoarthritis. Arthritis Care Res. 2010;62(8):1190-3.

6. Bhatia D. Current interventions in the management of Knee Osteoarthritis. J Pharm Bioallied Sci. 2013;5(1):30.

7. Mohammad JS, Chan SM, Rohit V, Shazia QJ. Role of complementary and alternative medicines in geriatric care: A mini review. Phcog Rev. 2014;8:81-7.

8. Changik Jo. Cost-of-illness studies: Concepts, scopes, and methods. Clin Mol Hepatol. 2014;20(4):327-37.

9. Elders MJ. The increasing impact of arthritis on public health. J Rheumatol. 2000;27(60):6-8.

10. Jaume PJ, Alba Ruiz Z. Socio-economic costs of osteoarthritis: A systematic review of cost-of-illness studies. Seminars of ArthRheu (Elseveir). 2015;44(5):531-41.

11. Sanjib S, Gerdtham UG. Cost of illness studies in osteoarthritis: a systematic literature review. Health econ rev. 2010;7(8):3150-95.

12. Feng $X$, Julian $T$, Kok-Yong $F$, Ngai-NL, Seng-Jin $Y$, Kuang $Y$, et al. A study on indirect and intangible costs for patients with knee osteoarthritis in singapore. Int Society Pharmaecon Outcomes Res. 2008;11(1):S84-S90.

13. Elena, Alexander M, William M, Sara A, Daniel H, Meghan E, et al. Life time risk and age of diagnosis of symptomatic knee osteoarthritis. Arthritis care res. 2013;65(5):703-11

14. David J, Deborah S, Emily C. The individual and socioeconomic impact of osteoarthritis. Nature Rev Rheumatol. 2014;10(7):437-41.
15. Leardini G, Salaffi F, Caporali R, Canesi B, Rovati L, Montanelli R. Direct and indirect cost of osteoarthritis of knee. Clinexper Rheumatol. 2004;22(6):699-706.

16. Mehdi Y, Abbas AA, Bahram S, Anoshirvan KJ, Somayeh F. Household health costs: Direct, Indirect and Intangible. Iran J Public Health. 2014;43(2):202-9.

17. Kim HS, Jinho L, Yoon J, Young B, Park B, In-Hyuk $\mathrm{Ha}$, et al. Association between knee osteoarthritis, cardiovascular risk factors and the Framingham Risk Score in South Koreans. PLoS One. 2016;11(10):e0165325.

18. Philippe B, Francois R, Laurent G, Jean-Noel D, Pierre B, Corinne E, et al. Annual cost of patients with osteoarthritis of the knee and hip in France. J Musculoskelet Pain. 2014; 10:1058-452.

19. Le TK, Montejano LB, Cao Z, Zhao Y, Ang D. Healthcare costs associated with osteoarthritis in US patients. Seminars ArthRheu. 2012;12(8):633-40.

20. Logan PA, Murphy A, Drummond AE, Bailey S, Radford KA, Gladman JR, et al. An investigation of the number and cost of assistive devices used by older people who had fallen and called a 999 ambulance. $\mathrm{Br} \mathrm{J}$ OccupTher. 2007;70(11):475-8.

21. Xin L, Monique AM, Aslam HA. The indirect costs of arthritis resulting from unemployment, reduced performance and occupational changes while at work. MediCare.2006;44(4):304-10.

22. Chen A, Gupte K, Akhtar K, Smith P, Cobb J. The global economic cost of osteoarthritis: How the UK compares. Arthritis. 2012; 2012.

Article History: Submission Date : 24-01-2018; Revised Date : 23-02-2018; Acceptance Date : 02-04-2018.

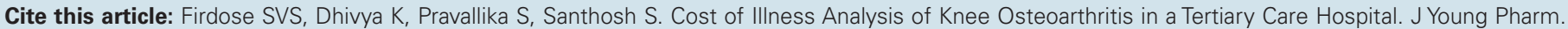
2018;10(3):322-5 